\title{
Therapeutic Ionizing Radiation to Head History Present
}

National Cancer Institute

\section{Source}

National Cancer Institute. Therapeutic lonizing Radiation to Head History Present. NCI

Thesaurus. Code C160162.

There is a history of the individual receiving therapeutic ionizing radiation to the head. 